\title{
Detection of changes in variance of oceanographic time-series using changepoint analysis
}

\author{
Rebecca Killick, Idris A. Eckley \\ Department of Mathematics and Statistics \\ Lancaster University \\ Lancaster \\ LA1 4YF \\ r.killick@lancs.ac.uk and i.eckley@lancs.ac.uk

\section{Philip Jonathan} \\ Shell Technology Centre Thornton \\ Production \\ P.O. Box 60 \\ P.O. Box 1 \\ Chester \\ United Kingdom. \\ philip.jonathan@shell.com
}

Kevin Ewans

Shell International Exploration and

2280 AB Rijswijk

The Netherlands.

kevin.ewans@shell.com

Corresponding author: Idris Eckley (+44 01524 593066) 


\begin{abstract}
Changepoint analysis is used to detect changes in variability within GOMOS hindcast time-series for significant wave heights of storm peak events across the Gulf of Mexico for the period 1900-2005. To detect a change in variance, the two-step procedure consists of (1) validating model assumptions per geographic location, followed by (2) application of a penalized likelihood changepoint algorithm. Results suggest that the most important changes in time-series variance occur in 1916 and 1933 at small clusters of boundary locations at which, in general, the variance reduces. No post-war changepoints are detected. The changepoint procedure can be readily applied to other environmental time-series.
\end{abstract}

Keywords: GOMOS; changepoint analysis; penalized likelihood; hindcast time series; significant wave height. 


\section{Introduction}

In the Gulf of Mexico, hindcasts such as GUMSHOE (Oceanweather (1990)) and GOMOS (Oceanweather (2005)) are frequently used in offshore design. . Motivated in part by recent extreme hurricane events, some authors have examined hindcasts more critically in recent years. For example, for the 1900-2005 GOMOS hindcast, Cooper \& Stear (2006) suggest that the intensity of some storms in the early 20th century is underestimated. A potential cause of underestimation is that meteorologists reconstructing early storms relied on coastal rather than offshore observations (see discussion in Section 4). There is a suspicion within the community that such hindcasts may be inhomogeneous in time. Cooper and Stear further suggest that, when pre-1950 storms are neglected, the six occurrences of category $4+$ hurricanes in 2004-5 (Charlie, Ivan, Dennis, Katrina, Rita, Wilma) do not "look as extraordinary". They also note the presence of a near-decadal cycle with peaks at around 1970, 1980, 1995 and 2004-5. As a result, the veracity of the pre-1950 portion of the data set is in doubt, and a number of practitioners currently only use post-1950 data in design (see for example Berek et al. (2007); American Petroleum Institute (2007)). Similar discussions also surround other databases, see for example Landsea et al. (2004).

Here, we investigate potential changes in variance (as opposed to change in mean or regression) for time series of significant wave height for the 1900-2005 GOMOS hindcast. At each of 2658 locations in the US sector of the Gulf of Mexico (see for example Figure 7(a)), we isolate storm peak significant wave height, $H_{S}^{s p}$, for 315 hurricane events (see Section 3 for further details of data). A time series plot for two typical locations, $\mathrm{X}$ and $\mathrm{Y}$, are shown in Figure 1(a) and 1(c) respectively. The maximum value of greater than $12 \mathrm{~m}$ occurs shortly before 1920 at both $\mathrm{X}$ and Y. Figure 1(a) and 1(c) show the mean profile for $H_{S}^{s p}$ across all spatial locations, together with a $95 \%$ confidence band for the mean. Variation at X and Y in time is consistent with Gulf mean variation, which interestingly shows an upward trend in $H_{S}^{s p}$ peaking before 1920. Figure 1(b) and 1(d) give corresponding histograms, which suggest a heavy-tailed distribution for $H_{S}^{s p}$.

Detecting changes in time series quickly and accurately is of interest to a wide range of disciplines, including bioinformatics (Lio \& Vannucci, 2000; Erdman \& Emerson, 2008), network traffic analysis (Kwon et al., 2006), climatology (Reeves et al., 2007), econometrics (Andrews, 1993; Perron \& Yabu, 2009) and engineering (Labeyrie, 1991; Willsky \& Jones, 1976). Given a sample of time series $\left\{y_{t}: t \in 1, \ldots, n\right\}$, a changepoint occurs if there exists a time $k$, where $1 \leq k \leq n-1$, such that the distributions of $\left\{y_{1}, \ldots, y_{k}\right\}$ and $\left\{y_{k+1}, \ldots, y_{n}\right\}$ are different with respect to some criterion. For example:

(a) Change in mean: in this case $y_{t}$ has mean $\mu_{t}=\left\{\begin{array}{ll}\mu_{1}, & t \leq k \\ \mu_{n}, & t \geq k+1\end{array}\right.$, where $\mu_{1} \neq \mu_{n}$. 
(b) Change in regression: Assume that $X_{t}$ and $Z_{t}$ are mutually independent and identically distributed sequences with $\mathbb{E}\left(Z_{t}\right)=0, \mathbb{E}\left(Z_{t}^{2}\right)=1, \beta_{1} \neq \beta_{n}$ and $\gamma_{1} \neq \gamma_{n}$. Then

$$
Y_{t}=\left\{\begin{array}{ll}
\beta_{1}+\gamma_{1} X_{t}+\sigma Z_{t}, & t \leq k \\
\beta_{n}+\gamma_{n} X_{t}+\sigma Z_{t}, & t \geq k+1
\end{array} .\right.
$$

(c) Change in variance: in this case $y_{t}$ has variance $\sigma_{t}^{2}=\left\{\begin{array}{ll}\sigma_{1}^{2}, & t \leq k \\ \sigma_{n}^{2}, & t \geq k+1\end{array}\right.$, where $\sigma_{1}^{2} \neq \sigma_{n}^{2}$.

We are typically interested in, (i) testing whether a change has occurred; (ii) estimating the location of the change; (iii) estimating the degree of change; (iv) providing a measure of confidence around the estimated changepoint (see Carlstein et al. (1994) and Chen \& Gupta (2000)).

This article uses changepoint analysis to identify potential occurrences (in time) of change in variance of $H_{S}^{s p}$, independently per spatial location for 2658 locations from GOMOS. Section 2 introduces the changepoint method and demonstrates its performance on simulated data. Section 3 applies the method to GOMOS $H_{S}^{s p}$. The paper concludes with a discussion and suggestions for future work.

\section{Detecting changes in variance}

Several changepoint methods to detect a change in variance exist, including cumulative sums of squares (Inclan \& Tiao, 1994), control charts (Hawkins et al., 2003), Bayesian posterior odds (Fearnhead, 2006) and penalized likelihood methods (Yao, 1988). We adopt a penalized likelihood approach using the Schwarz Information Criterion (SIC), proposed by Yao (1988).

Changepoint detection, per location in GOMOS, proceeds as a two-step estimation. In the first step, we estimate the most likely time point in the time series corresponding to a changepoint using maximum likelihood. Then, once the most likely time point has been estimated, we use a penalized likelihood deviance to estimate whether this potential changepoint is more consistent with random variation in the time series, or whether it should be considered as a true changepoint.

For a sequence $\left\{y_{i}\right\}_{i=1}^{n}$ of independent Normal random variables with parameters $\left\{\mu, \sigma_{i}\right\}_{i=1}^{n}$ respectively, we define the null hypothesis, $H_{0}$ and the alternative $H_{1}$, for a change in variance:

$$
\begin{aligned}
& H_{0}: \sigma_{1}=\sigma_{2}=\ldots=\sigma_{n}:=\sigma_{(1)} \\
& H_{1}: \sigma_{(1)}:=\sigma_{1}=\ldots=\sigma_{k} \neq \sigma_{k+1}=\ldots=\sigma_{n}:=\sigma_{(2)} .
\end{aligned}
$$


Here, $k$ is the unknown changepoint location, and $\mu, \sigma_{(1)}$ (and $\left.\sigma_{(2)}\right)$ are unknown parameters. Note the assumptions of constant mean and Normality throughout. The (negative) log-likelihood of the sample is given by $l=\sum_{i=1}^{n}\left(y_{i}-\mu\right)^{2} / \sigma_{i}^{2}+\sum_{i=1}^{n} \log 2 \pi \sigma_{i}$. To estimate $k$ of the changepoint, we calculate $l$ under the alternative hypothesis, $l\left(H_{1}\left(\hat{\mu}, \hat{\sigma}_{(1)}, \hat{\sigma}_{(2)}\right) ; k\right)$, independently for each $k \in(2: n-2)$. The estimated changepoint location $\hat{k}$ is defined as $\hat{k}=\arg \min _{k} l\left(H_{1}\left(\hat{\mu}, \hat{\sigma}_{(1)}, \hat{\sigma}_{(2)}\right) ; k\right)$.

Having estimated $\hat{k}$, the Schwarz penalized (negative) $\log$-likelihood $l^{*}$ is given by $l^{*}=l+\frac{q}{2} \log n$, where $q$ is the number of parameters in the models corresponding to the null hypothesis $(q=2)$ and the alternative $(q=3)$. The deviance statistic $\lambda=l^{*}\left(H_{1}\left(\hat{\mu}, \hat{\sigma}_{(1)}, \hat{\sigma}_{(2)}\right) ; \hat{k}\right)-l^{*}\left(H_{0}(\hat{\mu}, \hat{\sigma})\right)$ is used in a significance test. The null hypothesis (of no change at $\hat{k}$ ) is rejected when $\lambda>c_{\alpha}$ for Type I error rate $\alpha$ and critical value $c_{\alpha}$ (see Chen \& Gupta (2000)). Note that only the order of data is required for analysis; precise times of storm peak events are not incorporated.

To find multiple changepoints, we first apply the detection method to the whole time series. If no changepoint is detected we stop. Otherwise we partition the time series at the changepoint and apply the detection method to each partition independently. This procedure is iterated (see Algorithm 1) until no further changepoints are detected. As we are effectively re-analyzing the time series at each iteration the confidence level, $\alpha$, is no longer the overall Type I error for the analysis. An efficient variant of the algorithm as proposed by Inclan \& Tiao (1994) is used henceforth.

To quantify the performance of the changepoint detection method, we conduct a brief simulation study using time-series realizations of length 200. The first 100 iid data points were taken from a standard normal distribution, the second 100 data points were simulated from a normal distribution with mean 0 and variance $\sigma^{2}$. We considered 6 scenarios, each with different values of $\sigma: \sigma^{2}=1,1.25,1.5,2,3$ and 4 . The first scenario, corresponding to that of no changepoint, is used to estimate the false-positive rate. Remaining scenarios correspond to increasingly large changes in variance. We simulated 10,000 independent data sets for each scenario. Figure 2(a) shows response operating curves (ROC) of empirical Type I error against empirical power. As the ratio of variances increases, more power is available to detect changes correctly. Satisfactory power is obtained for variance ratios greater than 3. As Figure 2(b) demonstrates, for small changes of variance (variance ratio $\leq 2$ ), we cannot detect changes with confidence. The simulation study suggests that, when looking for a large change (variance ratio $\geq 3$ ), one should use a small $\alpha$ value to ensure that the true location of the change is detected. A more comprehensive related study has been conducted by Eckley et al. (2009). 


\section{GOMOS analysis}

We consider storm peak significant wave height values from the proprietary GOMOS Gulf of Mexico hindcast Study (Oceanweather, 2005), for the period September 1900 to September 2005 inclusive. Data are available (at 30-minute intervals) for a total of 4363 grid locations, at a grid spacing of 0.125 degrees in both latitude and longitude. Following the approach of Jonathan \& Ewans (2009), we retain 2658 "non-boundary" locations defined as follows. At a non-boundary location, it is possible to place a square box of dimensions $11 \times 0.125$ degrees centered at the location, such that all locations in the box belong to the full hindcast. In this way, non-boundary locations do not include coastal US regions and deep-water locations near Mexican water. 315 storm events were isolated, common to all non-boundary locations. For each storm period for each location, we isolated storm peak significant wave height, $H_{S}^{s p}$ for subsequent analysis.

For each of the 2658 spatial locations independently, we apply the changepoint method (as described in Section 2) to identify changes in variance with the time series for $H_{S}^{s p}$. However, as noted in Section 2 , we must first confirm that distributional assumptions underlying the changepoint method are not violated.

Box-Cox transformation to Normality: Figure 1(b) and 1(d) show that the distribution of $H_{S}^{s p}$ is heavy-tailed. Yet the changepoint method described is appropriate only for Normally distributed data. We cannot therefore proceed with changepoint analysis until data are transformed to approximate Normality using the Box-Cox transformation (Cunha \& Guedes Soares, 1999). Transformation of variables has been used in a number of contexts within the ocean engineering literature. For example, Ferreira \& Guedes-Soares (2002) use the Box-Cox transformation in their approach to model significant wave height and peak spectral period jointly. Eastoe \& Tawn (2009) use the transformation as a preprocessing step to model non-stationary time-series in extreme value analysis. For our analysis, the parameter $\lambda$ in the Box-Cox transform is selected for each location so that the distribution of the transformed data is approximately Normal. Following transformation, the corresponding time series plots and histograms (in Figure 3, for locations $\mathrm{X}$ and $\mathrm{Y}$, following the format of Figure 1) show that time series are now approximately Normal. A Kolmogorov-Smirnov test for Normality (Smirnov (1939)) confirms this. First-differencing to remove mean fluctuation: Figure 3(a) and 3(c) also show the Gulf mean trend in $H_{S}^{s p}$ following Box-Cox transformation. It is clear that, at our two typical locations $\mathrm{X}$ and $\mathrm{Y}$, and through the Gulf, the assumption of constant mean required for application of the changepoint method cannot be justified. However, after first-differencing the time series, de-trended data (Figure 4(a) and 4(c)) appear to have approximately constant mean in time at $\mathrm{X}$ and $\mathrm{Y}$ respectively; the mean profile is also constant in time. Moreover, data at X and Y are still approximately Normally distributed according to 
Figures 4(b) and 4(d). This is confirmed per location using a Kolmogorov-Smirnov test for Normality.

Changepoints detected: Having ensured that distributional assumptions are not violated, we proceed with changepoint analysis. Separate analyses were performed for each of 2658 GOMOS locations under consideration. A relatively small number of significant changepoints were detected. Interestingly times of occurrence of these changepoints show considerable clustering, as illustrated in Table 1. For different levels of nominal significance, $\alpha$, the table shows that changepoints are detected in October 1900, October 1905, November 1916, September 1925, December 1925 and September 1933. The most prevalent changepoint time is November 1916, for which changepoints are detected at 55 of the 2658 locations (at $\alpha=0.1$ ). Spatially, changepoints tend to occur at locations near the boundaries of our GOMOS region, as illustrated in Figures 7(a) (for $\alpha=0.1$ ) and 7(b) (for $\alpha=0.05$ ). In Figures 7(a) and 7(b), points are coloured and sized according to the estimated variance ratio $\left(\sigma_{(2)} / \sigma_{(1)}\right.$ or $\sigma_{(1)} / \sigma_{(2)}$, whichever is larger). The majority (71\%) of all changepoints detected correspond to decreases in variance.

As expected, the number of significant changepoints decreases as the confidence level for the changepoint test increases. The optimum confidence level depends on the ratio of pre to post changepoint variances (see Section 2). For detecting variance change ratios of between 2 and 3, $\alpha=0.1$ is optimal. At this level, changepoints were found at 101 individual spatial locations. To verify we examined pre to post change variances for each location. Of the 101 changepoints, 42 had a variance ratio between 2 and 3.

For detecting a change ratio of between 3 and $4, \alpha=0.05$ is appropriate. Figure 7 (b) shows locations where a change in variance was detected at the 0.05 level, points coloured black corresponding to ratios larger than 3. Ratios for the two black locations in Figure 7(b) are particularly large $(>>4)$. However, as there are no changes in variance found at the 0.01 level, we conclude that these changepoints are probably just due to random variation. This appears reasonable given the date of the change (October 1900).

\section{Discussion}

This paper discusses a penalized likelihood approach to detect changes in variance in oceanographic time-series data. The analysis requires two modifying steps to ensure that assumptions underlying the changepoint method are satisfied: (i) a Box-Cox transformation to Normality, followed by (ii) de-trending using first differences. A simulation study of the performance of the changepoint method suggests that a relatively large change in variance is required for detection with reasonable certainty.

Interestingly, the changepoints detected correspond to specific events in the period 1900-1933. Somewhat surprisingly, no changepoints are detected post 1933 for values of parameter $\alpha$ considered. This suggests that changes occurring pre 1933 are more significant than anything after 1933, and we might infer that the 
period 1934-2005 provides a relatively stable interval (in terms of variance) for design purposes. There is a solid body of knowledge concerning developments of observational and numerical modelling contributing to the hindcast post-war, which we might have anticipated would be reflected in changepoints in storm peak significant wave height from GOMOS. For instance: pre-1944, observations of hurricanes relied mainly on land-crossings and occasional ship reports. From 1945 to 1960, early aerial reconnaissance observations became available; in the period 1960 to 1990, improved areal reconnaissance (at increasing frequency) and satellite data could be used; post-1990, yet further improvements to observational and modelling methods occurred. Yet it appears from the current analysis that uncertainty of observational data pre 1934 might be of greater consequence in terms of variance of storm peak significant wave height. The apparent clustering of detected changepoints in time and space requires further explanation.

There is also interest in assessing the spatial variability of $H_{S}^{s p}$ in the Gulf of Mexico. To investigate this, and to demonstrate the flexibility of the changepoint method for variance established in section 2, we performed a brief study of local spatial variance in time. At each of the 2658 spatial locations, we calculated (per storm event) the variance, $s_{i j}^{2}$ of $H_{S}^{s p}$ across an $11 \times 11$ spatial neighbourhood centered at the location of interest for each storm $i=1, \ldots, 315$ and spatial location $j=1, \ldots, 2658$. We then seek to detect changes in variance (in time) of $\left\{s_{i j}^{2}\right\}_{i=1}^{315}$ for each $j=1, \ldots, 2658$. To satisfy distributional assumptions, Box-Cox transformation to Normality, followed by first-differencing, was necessary. Changepoints in October 1900 were the only significant events at all $\alpha$ levels.

One criticism of the current approach is the impact of data transformations to satisfy assumptions which underpin the changepoint method; in particular, whether both Box-Cox transformation and first differencing might alter inherent data structure and therefore the detection of changepoints. For identification of changepoint times this approach is valid as we preserve the order of the data. However, these are concerns if one wished to estimate values of variance changes. To address this concern, we might extend the current method to incorporate alternative (Weibull or Generalized Pareto) distributional assumptions. This would, we believe, be a valid and interesting extension to the changepoint literature, but would need accompanying numerical estimation of method performance under heavy-tailed assumptions. To avoid prior removal of non-constant mean, we might use Box-Cox transformed data (which pass the Normality test) and monitor for changes in both time series mean and variance. This is a more challenging problem, but ideas from Dynamic Linear Modelling suggest how this adaptation might be achieved. It would be useful to evaluate numerically the effects of Box-Cox transformation on the performance of the changepoint method and to consider alternative approaches, such as fractional differencing (Granger, 1986), for de-trending. We might consider fully non-parametric changepoint methods, applied directly to unadjusted data; however, there are currently few reliable approaches. Future work in this area should also 
include detection of changes in higher order structure or distributional quantiles; an extreme value analysis may find that the number of extreme events has changed over time. If the assumption of a parametric distribution proves complicated or contrived, a wavelet approach for detecting changes within non-stationary time series may be preferred.

\section{Acknowledgment}

The authors thank Jonathan Tawn and Paul Fearnhead for helpful discussions; Vince Cardone and Jim Stear for discussion on the non-homogeneity of the Gulf of Mexico hurricane data; Vince Cardone and Oceanweather for the use of the GOMOS data; and three anonymous referees for their constructive comments. R. Killick acknowledges financial support from Shell Research Limited and the Engineering and Physical Sciences Research Council (EPSRC). P. Jonathan and K. Ewans acknowledge financial support from Shell International Exploration Production.

\section{References}

American Petroleum Institute. 2007. Interim Guidance on Hurricane Conditions in the Gulf of Mexico.

Andrews, D. W. K. 1993. Tests for Parameter Instability and Structural-Change with Unknown ChangePoint. Econometrica, 61(4), 821-856.

Berek, E. P., Cooper, C. K., Driver, D. B., Heideman, J. C., Mitchell, D. A., Stear, J. D., \& Vogel, M. J. 2007. Development of Revised Gulf of Mexico Metocean Hurricane Conditions for Reference by API Recommended Practices. In: Proceedings of the 2007 Offshore Technology Conference. 18903.

Carlstein, E., Muller, H. G., \& Siegmund, D. (eds). 1994. Change-point Problems. Institute of Mathematical Statistics Lecture Notes.

Chen, J., \& Gupta, A. K. 2000. Parametric Statistical Change Point Analysis. Birkhauser, Boston.

Cooper, C., \& Stear, J. 2006. Hurricane Climate in the Gulf of Mexico. In: Proceedings of the 2006 Offshore Technology Conference. 18418.

Cunha, C., \& Guedes Soares, C. 1999. On the Choice of Data Transformation for Modelling Time Series of Significant Wave Height. Ocean Engineering, 26(6), 489-506.

Eastoe, E. F., \& Tawn, J. A. 2009. Modelling non-stationary extremes with application to surface level ozone. Journal Of The Royal Statistical Society Series C - Applied Statistics, 58, 25-45. 
Eckley, I. A., Fearnhead, P., \& Killick, R. 2009. Analysis of Changepoint Models. In: Barber, D., Cemgil, T., \& Chiappa, S. (eds), Bayesian Time Series Models. Cambridge University Press.

Erdman, C., \& Emerson, J. W. 2008. A fast Bayesian change point analysis for the segmentation of microarray data. Bioinformatics, 24, 2143-2148.

Fearnhead, P. 2006. Exact and efficient Bayesian inference for multiple changepoint problems. Statistical Computing, 16, 203-213.

Ferreira, J. A., \& Guedes-Soares, C. 2002. Modelling bivariate distributions of significant wave height and mean wave period. Applied Ocean Research, 21, 31-45.

Granger, C. W. J. 1986. Developments in the Study of Cointegrated Economic Variables. Oxford Bulletin of Exonomics \& Statistics, 48(3), 213-228.

Hawkins, D. M., Qiu, P. H., \& Kang, C. W. 2003. The changepoint model for statistical process control. Journal of Quality Technology, 35(4), 355-366.

Inclan, C., \& Tiao, G. C. 1994. Use of Cumulative Sums of Squares for Retrospective Detection of Changes of Variance. Journal of the American Statistical Association, 89(427), 913-923.

Jonathan, P., \& Ewans, K. 2009. A Spatio-Directional Model for Extreme Waves in the Gulf of Mexico. submitted to Journal of Offshore Mechanics and Arctic Engineering.

Kwon, D. W., Ko, K., Vannucci, M., Reddy, A. L. N., \& Kim, S. 2006. Wavelet methods for the detection of anomalies and their application to network traffic analysis. Quality and reliability engieneering international, 22, 953-969.

Labeyrie, J. 1991. Time Scales and Statistical Uncertainties in the Prediction of Extreme EnvironmentalConditions. Reliability Engineering 6 System Safety, 32(3), 243-266.

Landsea, C. W., Anderson, C., Charles, N., Clark, G., Dunion, J., Fernandez-Partagas, J., Hungerford, P., Neuman, C., \& Zimmer, M. 2004. The Atlantic hurricane database re-analysis project: Documentation for 1851-1910 alterations and additions to the HURDAT database. Pages 177-221 of: Hurricanes and Typhoons: Past, Present and Future. Columbia University Press.

Lio, P., \& Vannucci, M. 2000. Wavelet change-point prediction of transmembrane proteins. Bioinformatics, 16, 376-382.

Oceanweather. 1990. GUMSHOE: Gulf of Mexico Storm Hindcast of Extremes. 
Oceanweather. 2005. GOMOS-USA : Gulf of Mexico Oceanographic Study.

Perron, P., \& Yabu, Y. 2009. Testing for Shifts in Trend with an Integrated or Stationary Noise Component. Journal of Business and Economic Statistics, 27(3), 369-396.

Reeves, J., Chen, J., Wang, X. L., Lund, R., \& QiQi, L. 2007. A review and comparison of changepoint detection techniques for climate data. Journal of Applied Meteorology and Climatology, 6, 900-915.

Smirnov, V. 1939. Sue les Ecarts de la Courbe de Distribution Empirique. Recueil Mathematique (Matematiceskii Sbornik), 6, 3-26.

Willsky, A. S., \& Jones, H. L. 1976. Generalized Llikelihood Ratio Approach to Detection and Estimation of Jumps in Linear-Systems. IEEE: Trans. on Automatic Control, 21(1), 108-112.

Yao, Y. 1988. Estimating the number of change-points via Schwarz' criterion. Statistics and Probability Letters, 6, 181-189.

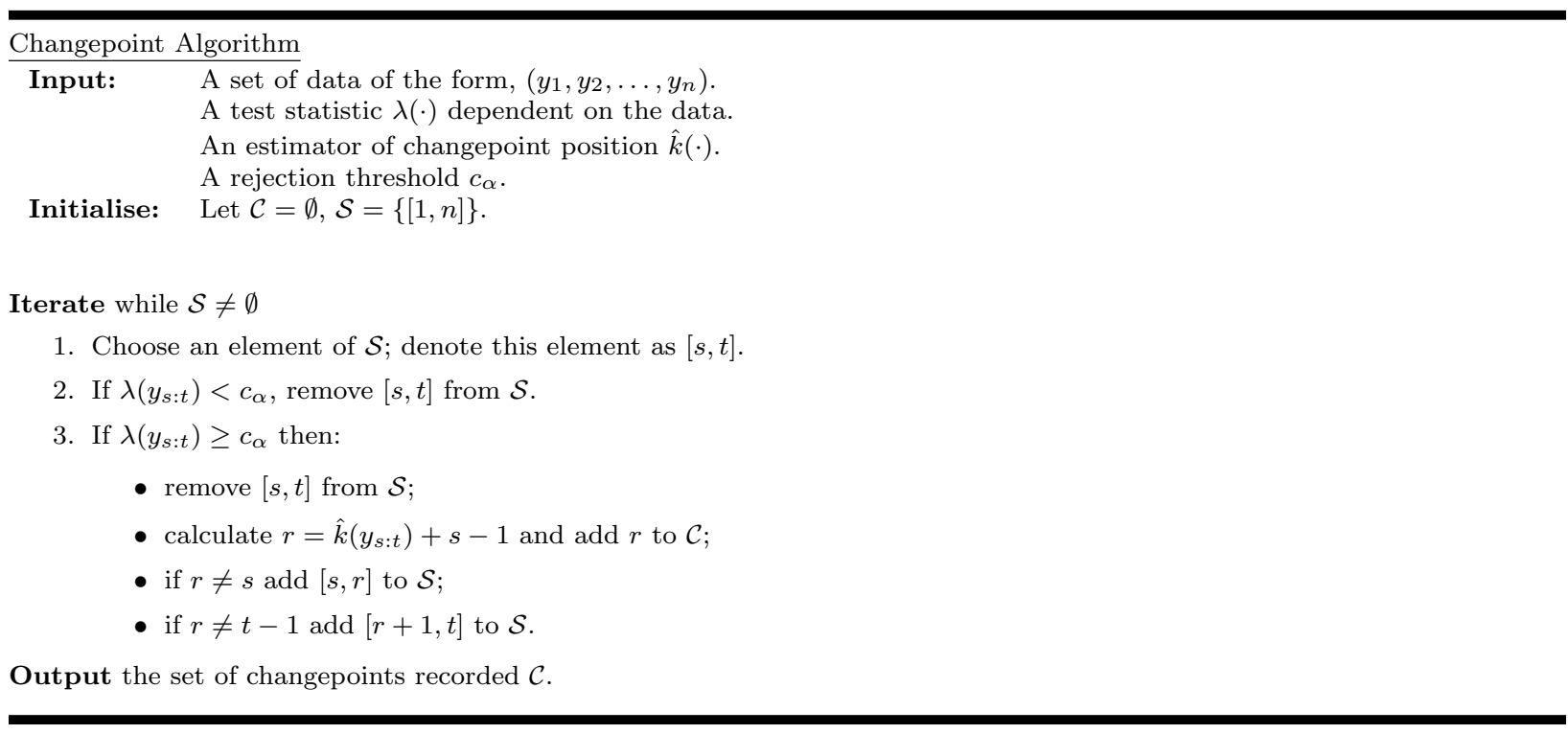

Algorithm 1: The Changepoint Algorithm. 


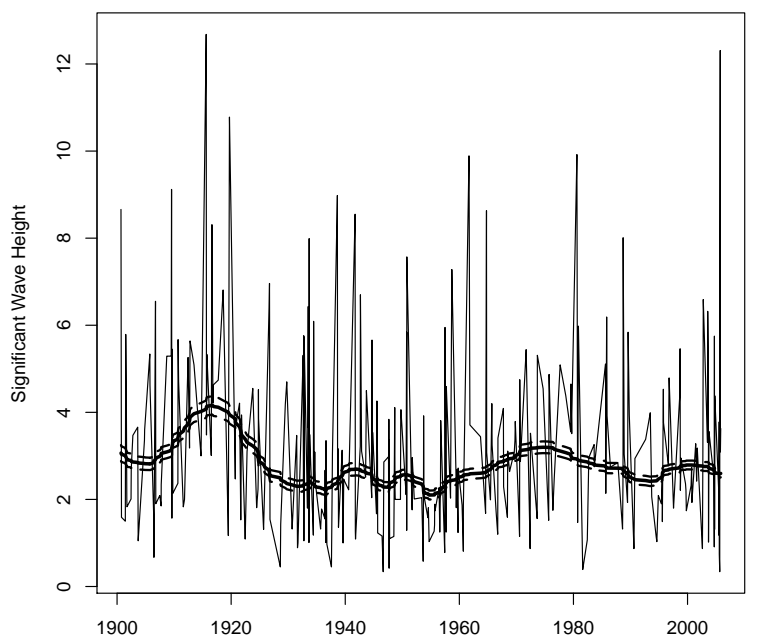

(a)

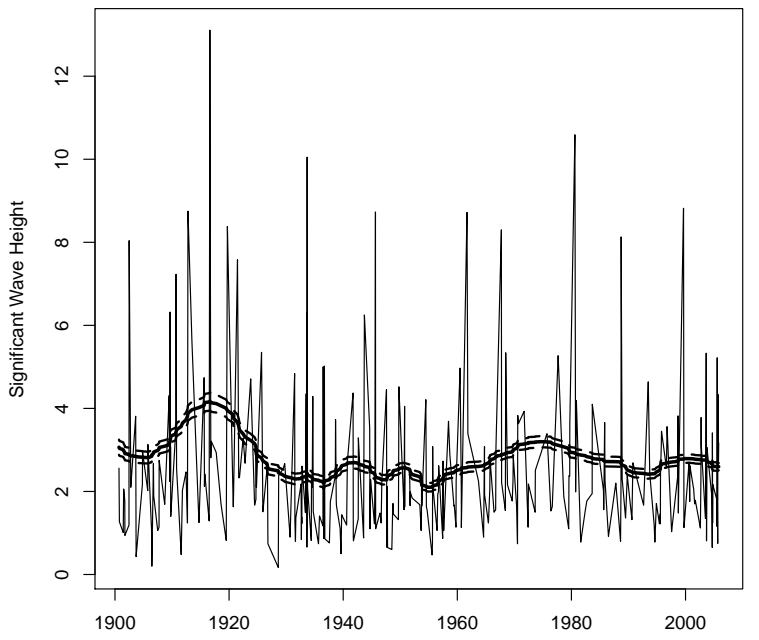

(c)

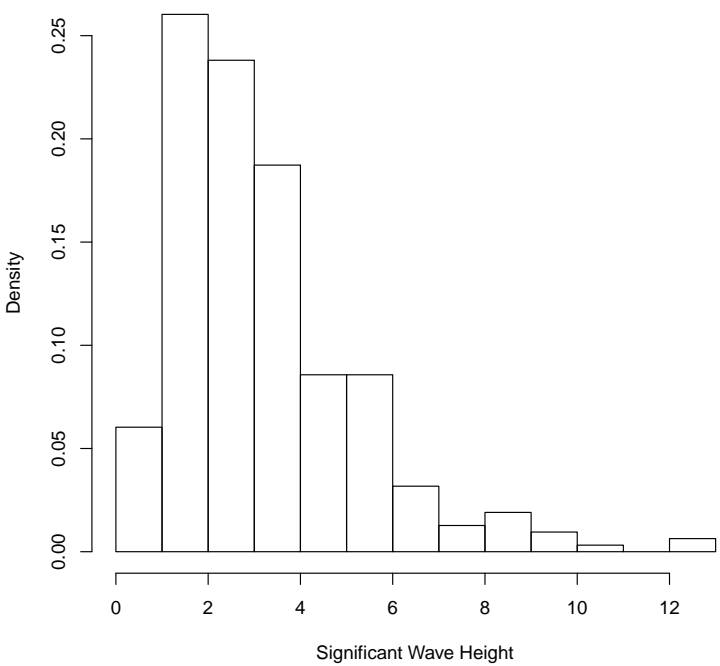

(b)

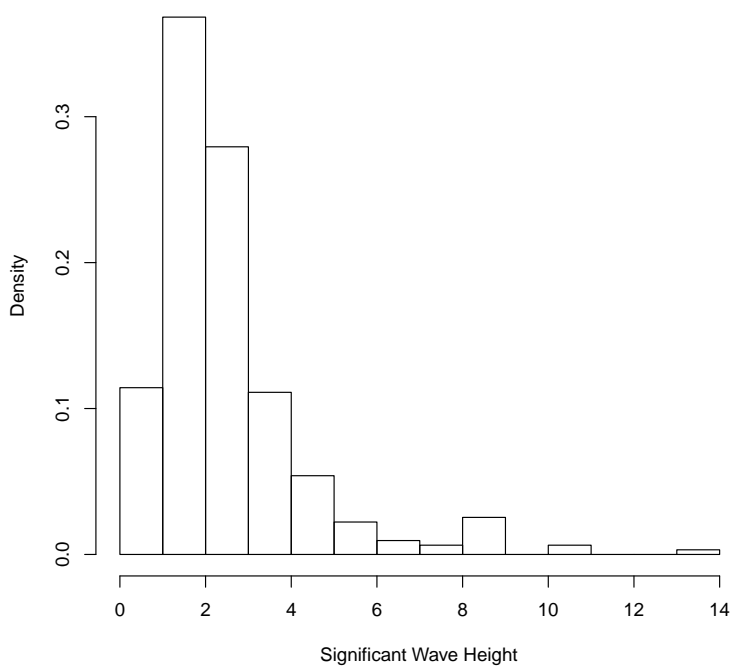

(d)

Figure 1: Time plots and histograms of $H_{S}^{s p}$ for two typical locations, X ((a) and (b)) and Y ((c) and (d)). A smoothed polynomial fit of the mean (across locations at a given time) and a $95 \%$ confidence band for the mean is also shown. 


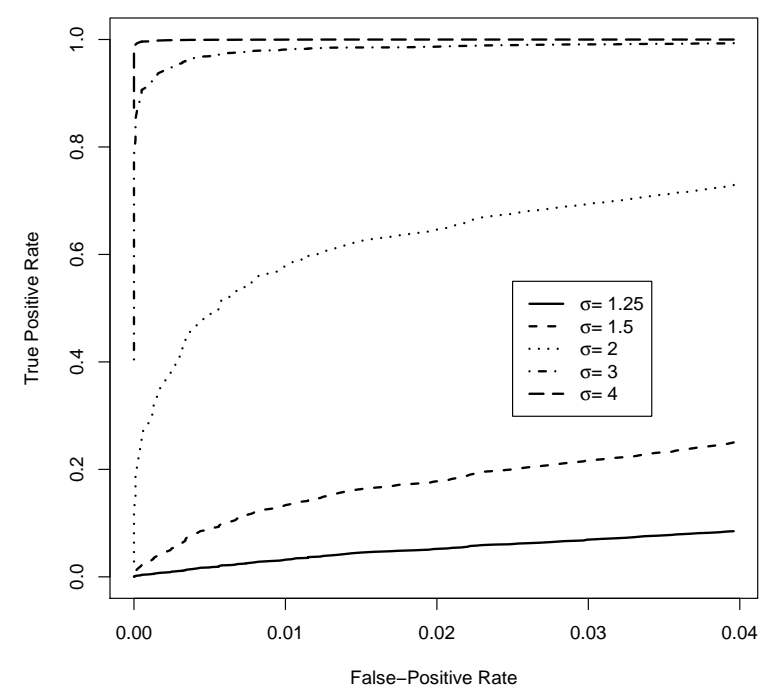

(a)

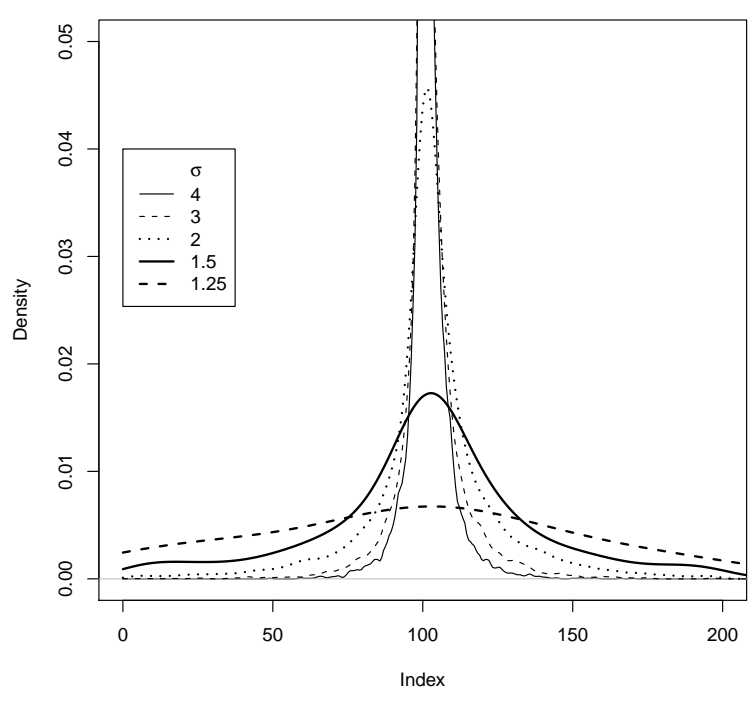

(b)

Figure 2: Simulation Results: (a) ROC curves for varing $\sigma$. (b) Body of density for detected location of changepoint (true value is 100) for fixed $\alpha=0.05$ and varying $\sigma$.

\begin{tabular}{|c||c|c|c|c|c|c|}
\hline \multicolumn{7}{|c|}{ First differenced Box-Cox Transformed Significant Wave Heights } \\
\hline \multicolumn{1}{|c||}{} & \multicolumn{4}{c|}{ No. of significant locations with storm at date (yyyy-mm) } \\
\hline$\alpha$ & $(1900-10)$ & $(1905-10)$ & $(1916-11)$ & $(1925-09)$ & $(1925-12)$ & $(1933-09)$ \\
\hline 0.1 & 3 & 3 & 55 & 5 & 10 & 25 \\
\hline 0.05 & 2 & - & 29 & - & - & 2 \\
\hline 0.01 & - & - & - & - & - & - \\
\hline
\end{tabular}

Table 1: Results of changepoint analysis on first differenced Box-Cox transformed significant wave heights at each of 2658 locations. 


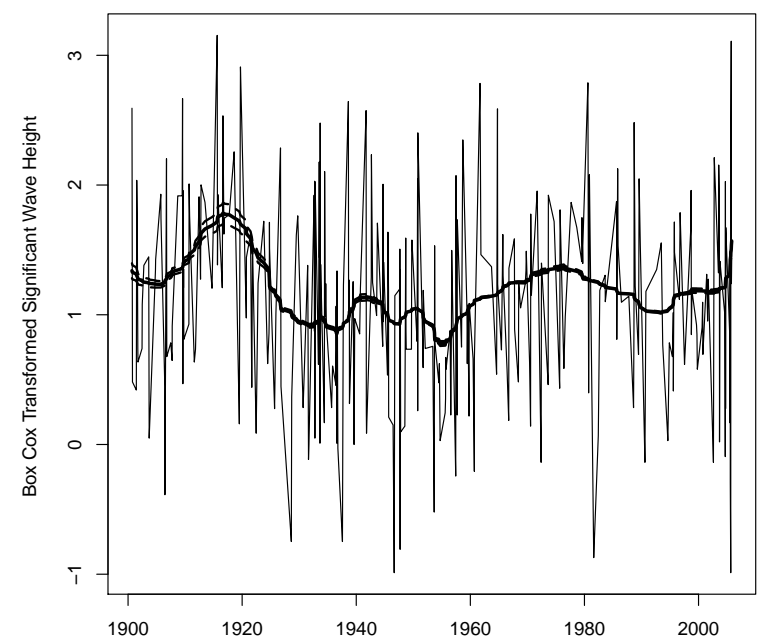

(a)

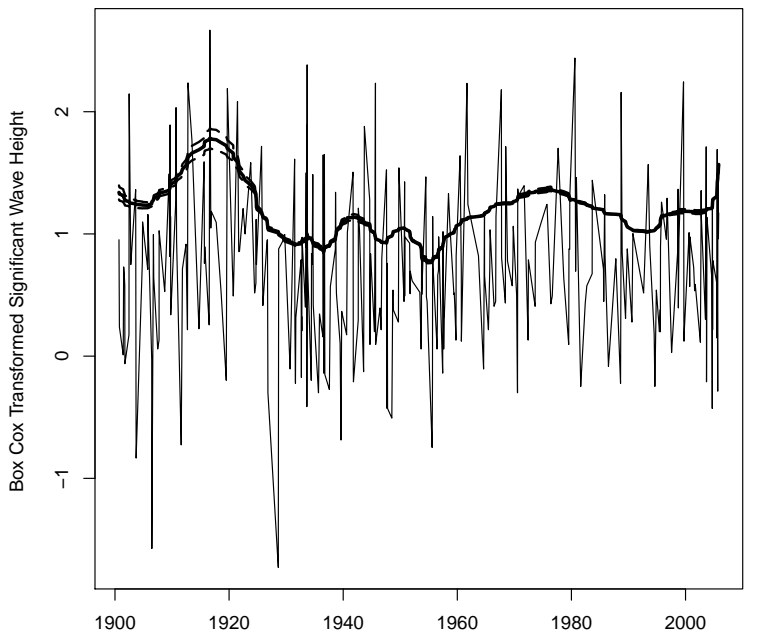

(c)

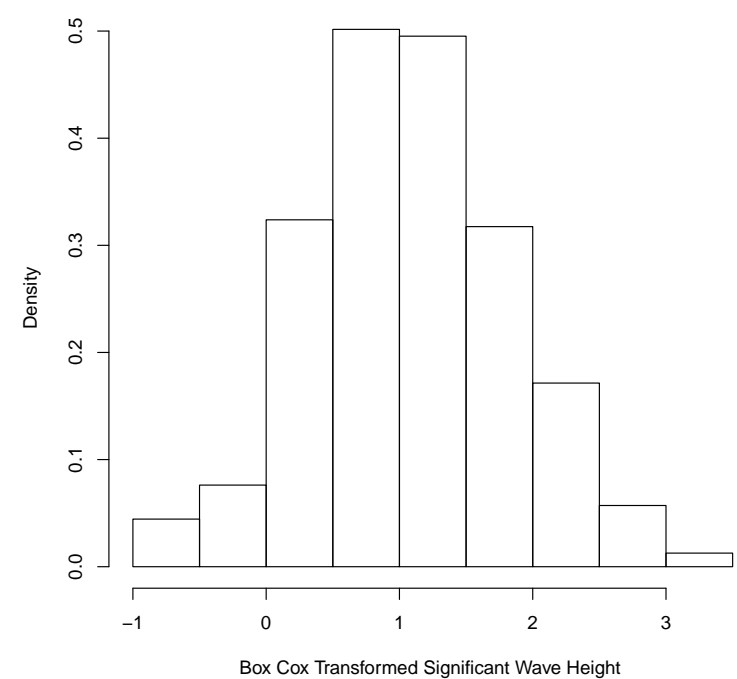

(b)

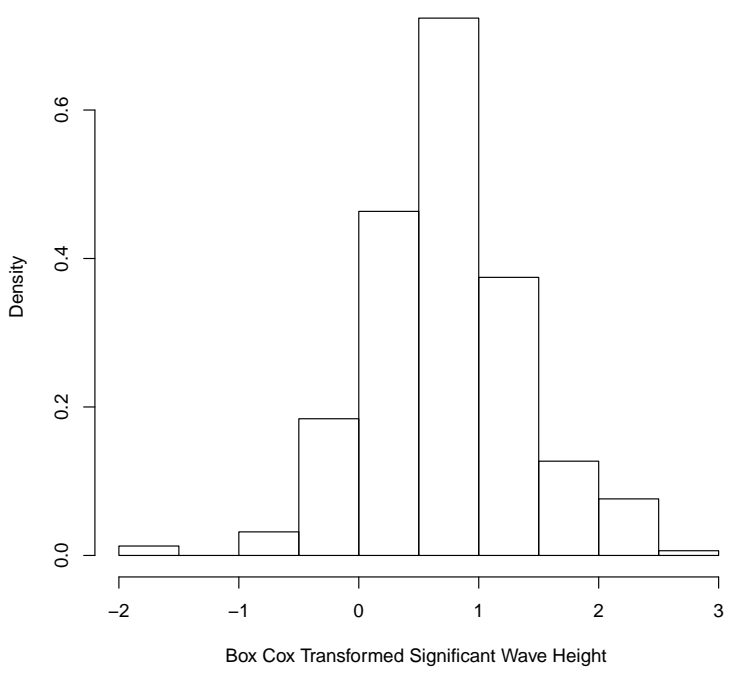

(d)

Figure 3: Time plots and histograms of Box-Cox transformed $H_{S}^{s p}$ for two typical locations, $\mathrm{X}((\mathrm{a})$ and (b)) and $\mathrm{Y}((\mathrm{c})$ and $(\mathrm{d}))$. A smoothed polynomial fit of the mean (across locations at a given time) and a $95 \%$ confidence band for the mean is also shown. 


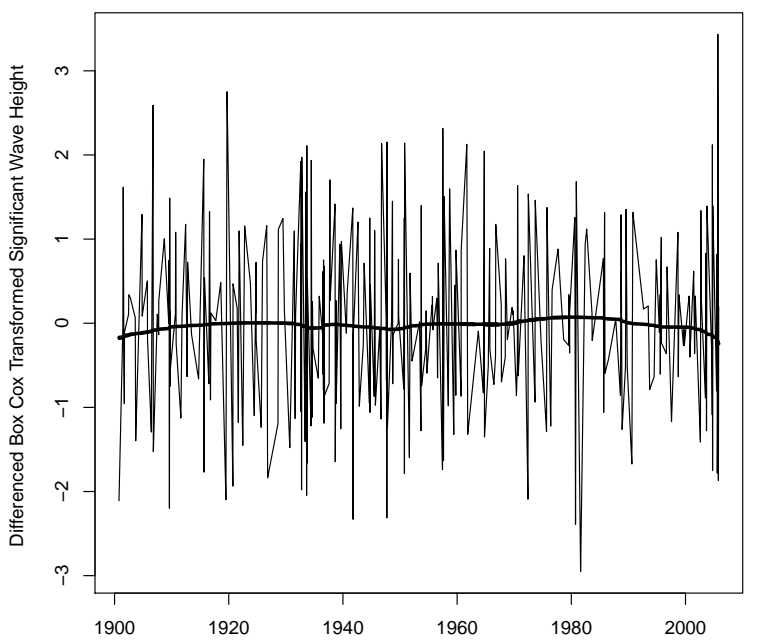

(a)

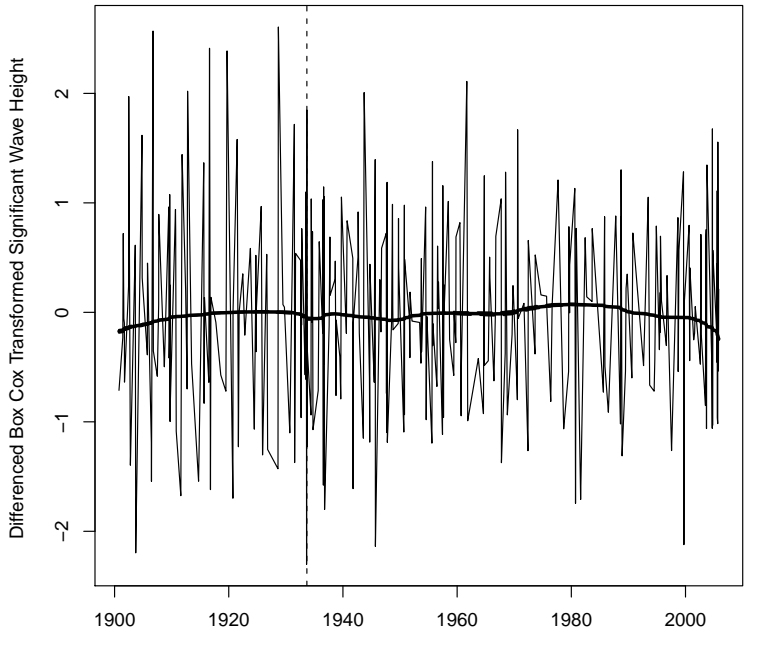

(c)

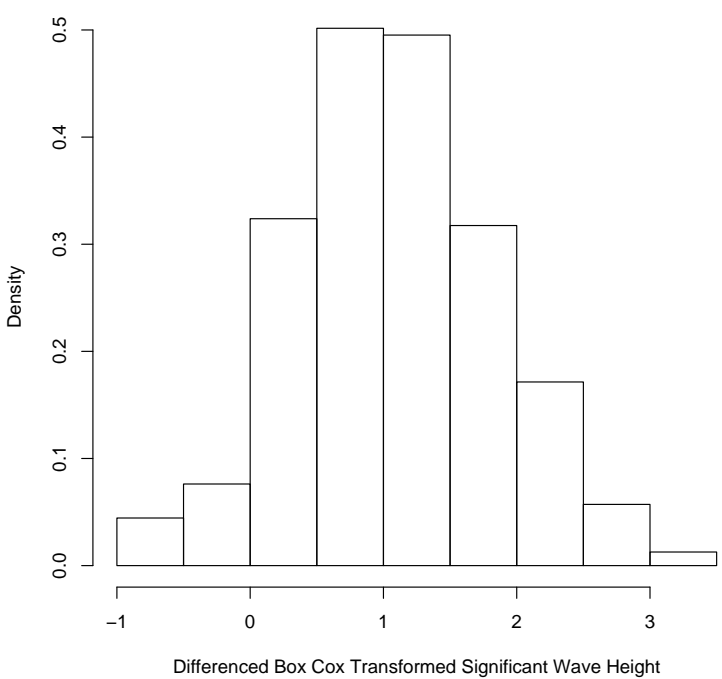

(b)

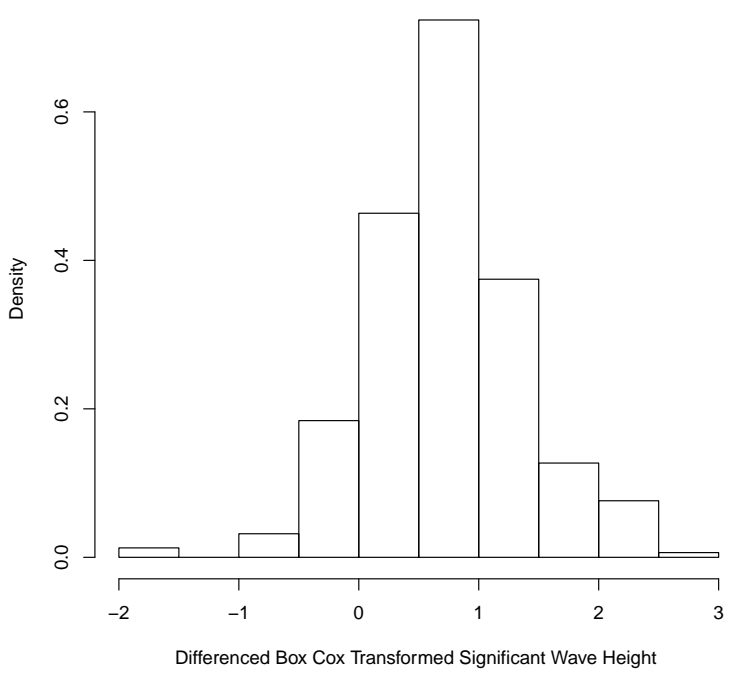

(d)

Figure 4: Time plots and histograms of the first differenced Box Cox transformed $H_{S}^{s p}$ for two typical locations, $\mathrm{X}((\mathrm{a})$ and $(\mathrm{b}))$ and $\mathrm{Y}((\mathrm{c})$ and $(\mathrm{d}))$. A smoothed polynomial fit of the mean (across locations at a given time) and a 95\% confidence band for the mean is also shown. The vertical dashed line in (c) at 1933 corresponds to the estimated time of a changepoint at location Y, see also Table 1. 


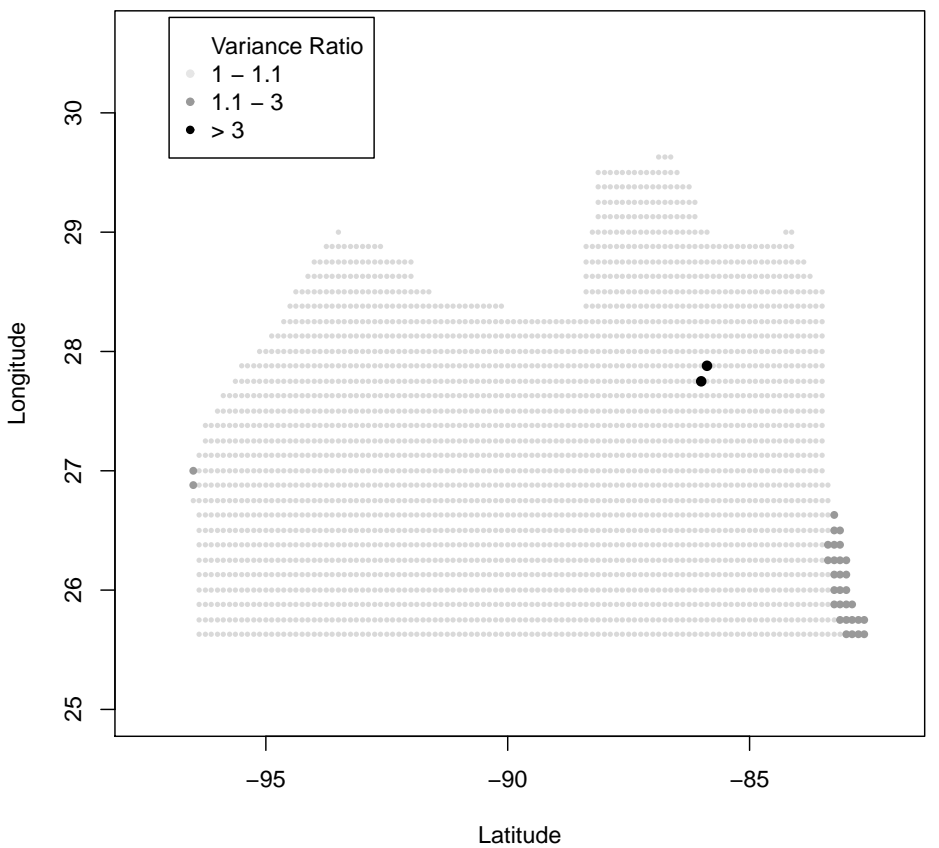

(a)

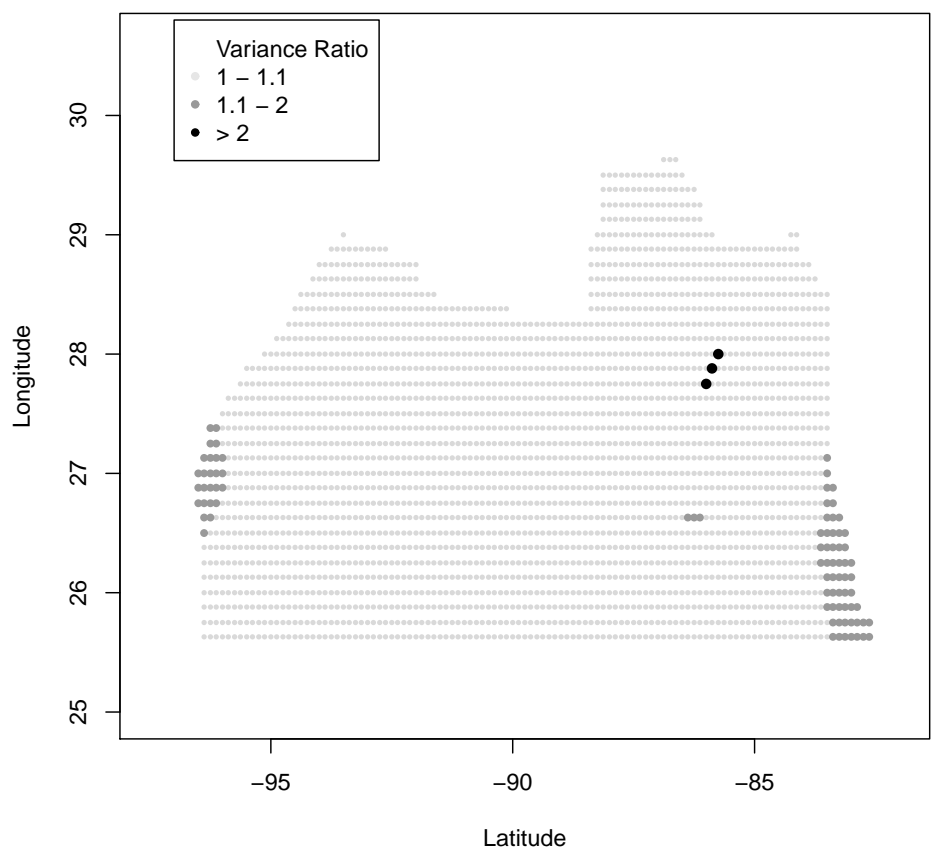

(b)

Figure 5: Map of the locations that have a change in variance at the (a) 0.1 and (b) $0.05 \alpha$ levels. 